Udine

\title{
SULLA PENETRAZIONE DEI PRESTITI ROMANZI NELLO SLOVENO
}

Com'è risaputo, la storia della penetrazione dei prestiti romanzi nello sloveno aialettale come in quello letterario è ancora quasi tutta da scrivere. O forse sarebbe meglio dire definire sotto i suoi molteplici ed anche problematici aspetti. Non ricorderò qui $i$ fondamentali lavori di vari studiosi quali K. Štrekelj, F. Šturm, lo stesso A. Grad ed altri ancora. Tutti hanno illuminato $i$ singoli ma anche frammentar aspetti di questo vasto ed irrisolto problema. Quelio che manea è peró un lavoro sistematico che tenga in considerazione soprattutto quei materiali che possano considerarsi validi per tutto il variegato mondo dialettale sloveno. I'unico tentativo in questo senso risale a ben trent'anni fa e non ebbe l'onore della stampa, anche se é facilmente consultabile. Alludiamo naturalmente alla opera del Mende. ${ }^{1}$

Gli studi finora apparsi non permettono dungue una visione organica ne tanto meno storicistica di questa penetrazione, tanto che appare ancora utile ripartire dalle constatazioni di F. Bezlaj: "zdi se, da je $v$ prvih stoletjih po naselitvi igral romanski adstrat bistveno važno vlogo ter ga je sele kasneje začel izpodrivati germanski. Vendar je nekaj specifičnih področij, $v$ katerih je romanski vpliv prevladoval tudi kasneje, posebno na prehodu med srednjim in novim vekom (...) Vseh romanskih plasti, s katerimi moramo računati na našem ozemlju, je približno štiri do pet, od vulgarne latinščine (...) do modernih izposojenk iz italijanščne, $k i$ jih je precej v naših zahođnih narečjih." 2

Che soprattutto i dialetti occidentali sloveni, a contatto diretto con il mondo romanzo, siano da considerarsi i maggiori mediatori dei romanismi è del tutto scontato ed ovvio. I mediatori dei romanismi diventano così $i$ dialetti ladini e veneti tra $i$ quali non vanno perô dimenticati gli ormai estinti tergestino e muglisano come spesso sottolineano nelle loro opere 
i dialettologi italiani ed in particolare G. B. Pellegrini e M. Doria. Un altro veicolo di mediazione dei romanismi è inoltre sicuramente l'istroromanzo 0 , se vogliamo, gli stessi dialetti dalmati italiani ecc.

Il problema "romanismi in sloveno" potrà essere affrontato con maggior sicurezza quando avremo a disposizione il tanto agognato dizionario storico sloveno del $16^{\circ}$ secolo, cosa che però non sembra imminente. Di enorme utilità potrà risultare gia il Registro della Bibbia del Dalmatin ${ }^{3}$. Mancandoci per ora troppi strumenti validi per uno studio diacronico, ci ripromettiamo oggi di esaminare il problema almeno dal punto di vista sincronico. Ci riferiamo a quei romanismi che vengono registrati da quello che a tutt'oggi rimane uno dei piú antichi vocabolari sloveni in generale e quelli italiano-sloveni in particolare.

La sorte di questo vocabolario è così anomala che merita certamente un breve excursus. Fu pubblicato a Udine presso G. B. Natolini - per inciso, primo tipografo friulano 4 nel 1607 con il titolo vocabolario/Italiano e schiauo / che contiene una breue instrut / tione per apprendere facilmente / detta lingua schiaua, le lox ordina / rie salutationi, con vn ragionamen / to famigliare per li viandanti./ Aggiuntovi anco infine il Pater / noster, 1'Aue Maria, il Credo, i Pre/ cetti di Dio, e della s. Chiesa, con al / cune lodi spirituali solite a cantarsi da / questi popoli nella maggior solennita / dell'anno. Raccolto da Fra Gregorio Alasia da sommaripa dell'ordine de' ser / ui della B. V. Maria.

L'autore era un servita piemontese, all'epoca in servizio a Duino nei pressi di Trieste presso il conte Raimondo della Torre e Valsassina. ${ }^{5}$ I libro fu una delle conseguenze dell'accordo che il conte aveva stupulato nel 1598 con l'ordine dei serviti onde arginare la penetrazione protestante (i protestanti erano stati appena scacciati). Fra' Gregorio, per dirla come il Caccamo nel Dizionario biografico degli Italiani "fu il primo sacerdote cattolico che si pose il problema dei testi in lingua slovena indispensabili alla cura delle anime, compilando, ad uso dei sacerdoti cattolici attivi tra gli sloveni, un dizionario...".6 
Un mistero totale avvolge la sorte e la fortuna del: dizionario (esemplari, diffusione, influssi...) per giù di duecento anni! L'unico esemplare a tutt'oggi conosciuto venne infatti scoperto ad un'asta pubblica a Vienna agli inizi del secolo scorso da Jernej Kopitar che vi appose la scritta opus valde rarum e si premuro di inviarlo a Lubiana per la celebre biblioteca di žiga Zois. ${ }^{7}$ E siamo ormai nel 1979 quando avviene la ristampa dell'opera, corredata questa volta da due saggi di accompagnamento che la collocano finalmente in una prospettiva storica. 8 . Questa riedizione offre infine a tutti gli studiosi la possibilita di valutare in giusta misura l'apporto dato dal servita piemontese alla lessicografia slovena. Sono infatti ben pochi gli studi precedenti dedicati al vocabolario dell'Alasia, anche se importanti. 9

Il problema più inquietante del vocabolario alasiano rimane il problema delle fonti di cui si servì o avrebbe potuto servirsi il frate piemontese. La valutazione fatta nel 1926 da Anton Breznik nel suo saggio slovenski slovarjilo (e quasi contemporaneamente da F. Kidričll ${ }^{l l}$ resta tuttora valida o, almeno, non è stata confutata dagli altri studiosi. Le fonti accertate dell'Alasia sarebbero cioè $i$ due dizionari di H. Megiser e, sempre per dirla con il Breznik, "neki hrvatski ustni vir" e ció per alcuni croatismi del tutto estranei al dialetto carsico occidentale che costituisce la base della conoscenza che l'Alasia ha della "Iingua schiaua". 12 che il servita abbia usato altre fonti protestanti e sicuramente la Bibbia del Dalmatin è naturalmente scontato. 13 Ai fini della nostra ricerca cio pero risulta poco rilevante. I romanismi tratti dall'Alasia dimostrano infatti da una parte la sua dipendenza dai due Megiser o, in senso lato, dagli altri autori protestanti, dall'altra invece registriamo i suoi "nuovi" romanismi (che peraltro potranno risultare già registrati nel dizionario del $16^{\circ} \mathrm{sec}$.!). Il confronto con il dizionario del Megiser risulta più agevole grazie a J. Stabej che ha riordinato il thesaurus polyglottus secondo il lemma sloveno. ${ }^{14} \mathrm{Il}$ confronto con 11 Megiser diventa infatti anche un confronto con gli autori protestanti in quanto vi si fa riferimento. 
I romanismi alasiani che trovano riscontro nel Megiser ${ }^{15}$

b a r c a (58), slov. barka, Megiser (6), Marg., Dict.;

b reg hes se (64) "calzoni", slov. -, Megiser (10) bregese, Reg., Dict.;

c h i b u 1 a (71) "cipolla", slov. čebula, Megiser (17), čebul, Reg.., Dict. Da ultimo il Bezlaj (I, 76) considera il termine un prestito dall'ital, ven. cebolla ovvero dal mated. zibolle, zebülle, zwibole, ambedue riconducibili al lat. caepula;

f. i g a (91) "fico", slov, figa, Megiser (33), Dict. Anche qui per il Bezlaj $(I, 128)$ è un prestito dall' ital. ven. figo ovvero dal mated. viga;

c a 1 a ma r e (64), slov. kalamar, Megiser (56) "atramentarium; calamarium", Dict. Dal lat. calamarius, si cfr. Bezlaj (II/ 11);

c a m b r a (65) "camera", slov. kamra e dial. kambra, Megiser (56) kamra, Marg., Boh., Reg., Dict. Dal lat. Camera attraverso il mated. kamer (Bezlaj II/14);

c a p pon (66) "cappone", slov. kopun e kapun, Megiser (64) kopun, Dict. Bezlaj (II/66) lo ricollega all'ital. cappone dal lat. cappo e seguendo il Battisti-Alessio (743) lo considera un antico relitto mediterraneo;

c m e t (76) "contadino", slov. kmet, Megiser (61) kmetič, Boh., Reg., Dict. La forma kmet apparirebbe soltanto nel $18^{\circ} \mathrm{sec}$. secondo il Bezlaj (II/47-48). Riconducibile comunque al lat. comes "accompagnatore, vassallo" (Bezlaj ibidem);

c o s t a g $n$ (67) "castagna", slov. kostanj, Megiser (65), Dict. Dall'ital. castagna, si veda Bezlaj (II/71);

ma n c a g n e (80) "difetto", slov. manjkanje, Megiser (82) manjkati, Boh., Dict. Bezlaj (II/166), seguendo lo Skok (II 1371), lo considera un prestito antico dal lat. volg.;

c hos $t i$ e $r$ (104) "oste", slov. ośtir, Megiser (121) ošter, Boh., Dict. Derivato da ošterija dal friul.ostarie o ital. ver. ostaria (Bezlaj II/Z60);

p r e d i g a t (147) "predicare", slov, pridigati, Megiser (150) predigovati e (145) predgati e predigati, Dict. Dal latino, ma probabilmente attraverso la mediazione tedesca;

v o g l i a (53) "appetito" e (79) "desiderio", slov. volja "desiderio", Megiser (217), Boh., Reg., Dict. Lo Skok (III, 614) 
lo riscontra già nel baltoslavo. Il significato "appetito" è dialettale e chiaramente influenzato dai vicini dialetti italiani;

v a g 1 i a t (184) "valere", slov: veljati, Megiser (212) veljati, Narg., Boh., Dict. Lo Skok (III, 563-4) sottolinea che fin dai tempi del Miklosich viene considerato un prestito dallo ital. vaglia "valuta, prezzo" dal lat. valere.

Romanismi alaşiani che non trovano riscontro nel Negiser (sono segnati con l'asterisco quelli ormai in disuso)

(t) a n g a $\mathrm{t}$ a $\mathrm{r}$ i c a (51) "ampollina", dal nome base greco e lat. gastra "vaso panciuto" attraverso la mediazione dell'ant. veneziano angastarola (Šavil 96). Si noti la sostituzione del suffisso. 'Cfr. anche Bezlaj ( $I, 140)$ s. V. gastara;

b u s s a $t$ (59) "baciare", slov. - , dialettalmente oggi bəšat.

c a s $t$ i g a $t$ (150) "punire" dal lat. ed ital. castigare (Battisti-Alessio $I / 79$ ) con la sostituzione del suff. per l'infinito. Nel Megiser troviamo soltanto kastiga (58);

(+) C h i a i p o l a (96) "gabbia", slov. - ; dal friul. sciaipole (Pirona 964), passato poi a tajba (Bezlaj I/72) probabilmente attraverso il friul. s'ciaipie (Pirona 964). Si veda a questo proposito soprattutto il Pellegrini, Contatti (775) per i passaggi dal lat. cavea > caiba > $k^{\prime} a i b a$;

c 1 ○ c i a (70) "chioccia", slov. kloča, kloka, kvočka (Pleteršnik I, 411). Il termine carsico è chiaramente influenzato dal friul. clocie (Pirona 161-2) a anche ts. clocia. Da ultimo Doria (VII, 96) sottolinea il doppio ladinismo (mantenimento del gruppo $c l$ e $c+a>c i a)$;

chiot (191) "zoppo" e c hi o $t$ a $t$ (191) "zoppicare", per il Bezlaj (I, 87) čot "sepast" dall'ital. ciotto, dialettalmente zoto. Lo Šturm (ČJKZ VI, 79 e CJJKZ VII, 50) pensa soprattutto all'ant. friul. * cot ovvero * chod da claudum;

(+) d e p e n a $t$ (52) "annullare", slov. -, isolato italianismo;

e $l$ e m e $\mathrm{n}$ (85) "elemento", slov. element, termine dotto dal lat. elementum con la probabile mediazione tedesca; 
f a z zo let (89) "fazzoletto", per lo slov. faconetel si veda il Bezlaj (I, 126), slov robec. Dal ven. fazzoleto o friul. fazzolet. La forma ancora vitale è fatou dal friul. faz$z \hat{u} I$.. (Pirona 303) ovvero dall'ant. friul. facul come pensa lo Šturm (CJKZ VI, 64) che considera lo c dei prestiti nello sloveno di origine prevalentemente friulana;

$f i$ tat (48) "affittare", slov. najem, ma dialettalmente vivo fìt, si veda Bezlaj (I, 129). Nei dialetti carsici fat con il derivato $f t j j r t$ con l'aggiunta di un suff. tipico di influenza germanica;

g h e r d e 11 e (99) "gradella", slov. gradelj "raženj" (Bezlaj I, 168) dal lat. craticula attraverso il ven. gradella o meglio friul. gardela (Pirona 405);

(+) gre s.t: (49) "agresto", slov. - (ma si cfr. grest "divji, surov": in Bezlaj II, 175):. Dal lat. agrestris (Battisti-Alessio I, 95) attraverso la mediazione friul. agrest (Pirona 7) e grest (Pirona 404), presente anche nel ven. agresto e gresta "uva acerba" (Boerio 317);

ma n i g. a u l t (62) "boia", slov. - . Nel Megiser (82) troviamo maningolda: che sta erroneamente per manigold "carnifex". Il significato "boia" è antico e dialettale e soltanto nel $16^{\circ}$ sec. in Ariosto e Berni diventa sinonimo di "furfante, briccone", cfr. Battisti-Alessio III, 2349 ;

$\mathrm{n}$ u $\mathrm{n}$ e $\mathrm{z}$ (136) "padrino", slov. - In resiamo nun "padrino di battesimo" accanto a nura: "padrina di battesimo. Il significato si mantiene solo nel sardo (nonnu, nonna), altrove passa a "nunno, nonna". Dal lat. nonnus, cfr. Bezlaj II, 230;

oz z a t (46) "aceto", slov. kis ma dial. ocet. La base e il lat.acetum ma Bezlaj (II, 239) pensa ad una mediazione del got. akcit ovvero a un antico rom. acitum;

p a g a n s t u o: (10.5) "idolatria", slov. poganstvo.. Il nome base pogan trova peró riscontro nel Megiser (133), Reg., Dict. Dal lat. paganus che deriva da pagus, cfr. Skok II, 694; p a u r i e (99) "granciporri", slov. - . Ancora vitalissimo nei dialetti carsici, evidente prestito dal ven. pavor, pavour;

p e s t a $t$ (64) 'calcare", molto vivo nei dialetti occ., 
slov. - , dall'ital. pestare con la gia notata sostituzione del suff. straniero con uno indigeno;

p r o c s s $i$ a (149) "processione", slov procesija;

r a d i c h (71) "cicoria", slov, radic, dal lat. radiculus, ital. radicchio attraverso la mediazione del ven. ts. radicio, si veda 10 skok III, 96. La forma goriziana redrik risente invece l'influsso del friul. radrîc (Pirona 841);

$(+)$ i $\circ$ i a (65) "campagna", slov. II pleteršnik (II, 435) registra soltanto i significati di "roggia; insieme di piante acquatiche". Il significato "campagna" dell'Alasia non trova riscontro neanche nel friul roe, roja, roje (Pirona 893) da cui evidentemente deriva. Anche il Miklosich, ET $278 \mathrm{~s} . \mathrm{v}$. roje riporta soltanto "muhlgang; mesta, kjer ob deževju voda vstaja kvišku iz taI";

s $t$ e $n$ a (89) "fatica", st $t n t$ a $t$ (89) "faticare", slov. - Attraverso la mediazione dell'ital. stentare o ven. stentar, dal lat. extentare, si veda lo skok (III, 413). Ancora vitale nei dialetti carsici come stantjarat dove notiamo un doppio suffisso per l'infinito, jar tat;

stregliat (177) "stregliare", slov.strgljati "čohati". Secondo lo skok (III, 417) è un derivato da štrigoj "česagija, čsalo", relitto lessicale dalmatoromanzo e istroromanzo dal lat. strigilis che in ital darà striglia:

s u b l a (117). "lesina". slov. - , dal friul. suble accanto a sùbule, sùbula (Pirona 1164), ven. subia (Boerio 721) tutti dal lat. subula;

va n d i m a (185) "vendemmia", slov. dial. bendima: dal friul. vendème (Pirona 1246) ovvero dall'istrorom. vandime, si cfr. Bezlaj I, 17, Mende 42, Miklosich, ET 9, Skok I, 106-7. Secondo 1o Šturm (CJKZ VII, 22) la $b$ è secondaria e proviene dal $v$;

$z$ e z o u (137) "palletta del fuoco", sloveno žeželj.Già il Pleters̆nik (II, 96.0) 10 confronta con l'ital. sessola anche se non è da escludere $l^{\prime}$ influsso del bav. schüssel (s. v. žežel, žèžoly.

Tra $i$ casi particolari vanno infine annoverati fratar (181) 
al quale I'Alasia attribuisce il solo significato di "traditore" accanto a fratat "tradire". Fratar appare gia nei due Megiser ma con il solo significato di "monachus". E un chiaro riferimento alle fonti protestanti?

Per il significato "campanile" si usa ancora a Duino e dintorni il termine $t$ e $r$ (65). Si è pensato ad una forma corrotta dal rom. torre. Poichè però nei dialetti vicini si usa tuttora il germanesimo turn da turm è possibile che la n finale sia caduta mentre la vocale accentata ha subito un esito imprevisto, del resto riscontrabile in altri casi per l'aspetto particolare che il dialetto di Duino e dintorni presenta nel vocalismo. 16

Concludendo, risultano decisamente interessanti quei romanismi che non trovano riscontro nei dizionari precedenti e che il Sommaripa, per quanto ne sappiamo finora, registra per la prima volta. Ed anche quelli ormai in disuso si dimostrano utili e significativi nello studio e nell'analisi della penetrazione dei romanismi cosi nello sloveno dialettale come in quello letterario.

1 M. Mende, Romanische Lehnwörter im Slovenischen, ed. dattiloscritta in "Slavisches Seminar der Freien Universität Berlin", senza I'indicazione dell'anno. Si tratta comunque della dissertazione inaugurale del Mende che risale al 1950. L'opera è consultabile anche nell'Istituto di Lingüe e letterature dell'Europa orientale dell'Università di Udine.

2 Cfr. F. Bezlaj, Eseji o slovenskem jeziku (Ljubljana: 1957), pp. $32-33$.

3 Sembra che il lavoro sia quasi terminato e sarà consultabile presso la sezione "Historični slovarji" delia sAzU.

4 G. B. Natolini (j551-1609) aprì la prima tipögrafia ad Udine nel 1592. Stampò allmeno l06 tra volumi ed opuscoli. A portare l'invenzione del Gutenberg nel friuli era stato Gerardo. di Lisa che operò nel 1480 a Cividale e nel 1484 ad Udine. L'esempio precoce di cerardo non ebbe peró un seguito continuato fino al Natolini.

Si veda, da ultima, N..Godini, per la collocazione di G. B. Natolini, primo tipografo friulano, in "Ce fastu?" LIX (Udine 1983, I), pp: 23-28 con I'indicazione della ricca bibliografia sulla stampa nel friuli-Venezia Giulia di G. Comelli.

5 Fra' Gregorio Alasia da sommaripa, al secolo Alessandro, era nato presso Cuneo nel 1578. Arrivo a Duino nel l60I e vi celebro anche la sua prima messa. Ripartì per sommaripa nel I609 oppure 1612. Morì a Roma nel 1626. Fu uno dei figli del 
conte, Raimondino alias Mattia della Torre ad incitarlo, incoraggiarlo ed aiutarlo nello studio della lingua slovena. E probabilmente fu lo stesso conte Mattias a volere la stampa dell'opera. La tiratura delle edizioni natoliniane ci rimane ignota, ma la stessa stampa del manuale dimostra che all' epoca dovevano essere ben numerosi i sacerdoti italiani che, disseminati in vari luoghi lungo il confine italo-sloveno, abbisognavano di un manuale che $1 i$ aiutasse nell'opera propagandistica della fede tra gli sloveni. per inciso, il conte Raimondo VI aveva assunto per eredita i possedimenti dei signori di Duino, compresi all'incirca.nel triangolo carsico Devin-opatje selo-Tomaj (Duino-opacchiasella-Tomadio) con l'aggiunta perd di numerosi castelli tra $i$ quali quelli di Senožeče (Senosecchia) e Fiume.

6 Cfx. Dizionario biografico degli Italiani (Roma 1960...), p. 585.

7 Lo riferisce lo stesso J. Kopitar in Hesychii glossographi discipulus (wien 1840) a p. 47: "(...) Somaripa capucini Dittionario Italiano et Schiavo, Udine 1607, 12., a nobis praeter exspectationem emptum in auctione publica. Vindobonae et e nostra donatione cum reliqua bibliotheca zoisiana nunc in Bibliotheca Lycei Labac. servatum. "L'anno del ritrovamento rimane però sconosciuto. Poichè lo zois morì nel 1819 si potrebbe supporre che l'acquisto del kopitar sia avvenuto prima.

8 La riedizione porta il titolo Gregorio Alasia da sommaripa: Vocabolario Italiano-Sloveno, altri testi italiano-sloveni e testi sloveni (Lubiana-Duino Aurisina (Trieste) 1979). Si tratta di un'edizione bilingue, italiano-slovena. C'è la riproduzione in fac simile del manuale alasiano (pp. 1-225) a cui seguono i saggi di L. Legiša, Gregorio Alasia da Sommaripa ed il suo vocabolario (pp. 228-285) e di B. Gerlanc, La nuova edizione di Alasia (pp. 286-303).

9 si veda $v$. oblak, Doneski $k$ historični dialektoloqiji, in. "LMS" (1891), pp. 66-130; F. Kidrič, Fra' Gregorio Alasia di Sommaripa, in "Ljubljanski zvon" (1924) pp. I02-110; $A$. Breznik, Slovenski slovarji, in "RDHV" III (1926), pp..IIO-116. Va segnalata però anche un'opera che non ebbe l'onore della stampa (reperibile alla NUK di. Lubiana ed anche presso il già citato. Istituto LLOE dell'Universita di Udine). Si tratta della tesi di laurea difesa nell'A. A. 1960-1 all' Università di Roma da G. Savli, Contributi alla conoscenza del Vocabolario italiano e schiavo di Alasia da Sommaripa (pp. 116).

10 Cfr. nota 9.

11 Ibidem.

12 Ibidem.

13 Com'è arcinoto, la traduzione della Bibbia del Dalmatin fu l'unico testo protestante ad essere tollerato. Ma nella biblioteca dei conti di Duino c'erano sicuramente altri testi protestanti. Lo dimostra lo stesso uso che l'Alasia fa dei due dizionari del Megiser. 
14 Il titolo completo è Hieronymus Megiser, Thesauxus polyglottus. Iz njega je slovensko besedje z latinskimi in nemskimi pomeni za slovensko-latinsko-nemski slovar izpisal in uredil Jože stabej. (Ljubljana 1977).

H. Megiser (1554-1619) fu autore di almeno 40 opere a stampa e 30 manoscritti. Per gli sloveni sono fondamentali però quei dizionari, il già citato Thesaurus del 1603 ed il Dictionarium quatuor linguarum del 1592, dove, per la prima volta nella storia, lo sloveno viene considerato allo stesso livello dei ben più gloriosi latino, tedesco ed italiano.

15. Va tenuto presente che i romanismi sono registrati nella grafia alasiana, cioè una grafia italianeggiante che creo al servita numerose difficoltà ed incertezze. Accanto e riportata la stessa voce nella forma e grafia slovene letteraria.

16 Si veda a questo proposito soprattutto I'opera dattiloscritta di G. Savli.

\section{Bibliografia}

BATTISTI-ALESSIO = C. BATTISTI-G. ALESSIO, Dizionario etimologico italiano I-V (Firenze 1975)

BEZLAJ = F. BEZLAJ, Etimološki slovar slovenskega jezika I/A-J (Ljubljana 1977), II/K-O (Ljubljana 1982).

BOERIO = G. BOERIO, Dizionario del dialetto veneziano (Venezia 1856)

BOH. = A. BOHORIĆ, Arcticae horulae … (1584)

DORIA $=$ M. DORIA, Dizionario del dialetto triestino (A-ingradela), non ancora apparso in volume. pubblicato inizialmente sulla rivista triestina "La Bora" che pero. dal 1981 non esce più.

DIC. = H. MEGISER, Dictionarium quatuor Iinguarum (1592)

MARG. = Appunti marginali nella Bibbia del Dalmatin (1584)

MIKLOSICH, ET = F. MIKLOSICH, Etymologisches wörterbuch der slavischen Sprachen (Wien 1886)

PELLEGRINI, Contatti = C. PELLEGRINI, Contatti linguistici slavofriulani in "studi linguistici in onore di vittorio Pisani "II (Brescia 1969), pp. 761-776

PLETERŠNIK = M. PLETERŠNIK, Slovensko-nemški SlOVar (1894-5). Ristampa Ljubljana 1974 .

REC. = Registro della Bibbia del Dalmatin.

SKOK $=P$. SKOK, Etimologijski riečnik hrvatskoga ili srpskoga jezika I-IV (Zagreb 1971-1974)

ŠVLI = G. ŠVLI, op. cit.

STURM CJJKZ VI = F. STURM, Refleksi romanskih palataliziranih konzonantov $v$ slovenskih izposojenkah in CJKZ VI (Ljubljana 1927), pp. 45-85. 
ŠTURM ¿JKZ VII = F. ŠTURM, ROmanska lenizacija medvokaličnih konzonantov in njen pomen za presojo romanskeqa eIementa $v$ slovenšcini in CJKZ VII (Ljubljana 1928), pp. $21-46$.

Povzetek

O VDORU ROMANSKIH JEZIKOVNIH PRVIN V SLOVENSЦ INO

Vdor romanizmov $v$ tkivo slovenskega knjižnega jezika oz. zgodovino tega vdora šele raziskujemo. Pri romanskih izposojenkah moramo namrec racunati do pet plasti, ki so pa včasih bolj težko opredeljive. $z$ objavo gradiva iz Dalmatinovega Registra bo seveda to preučevanje znatno lažje.

$\checkmark$ tem prispevku si avtorica prizadeva preučiti romanizme, $k i$ nam jih nudi Alasijev slovar iz leta l607. Primerja jih s tistimi, $k i$ jih je že registriral H. Megiser v svojem Thesaurus polyglottus oz. s tistimi, ki so že prisotni pri ostalih protestantskih piscih. Ugotavlja, da Alasia prinaša nekaj novih. Pomembni se ji zdijo tudi tisti romanizmi, ki so sicer že izumrli, a nam kot vsako možno gradivo tudi po svoje osvetljujejo proces, $k i$ nas zanima. Alasijevo.gradivo je še posebno zanimivo, ker je odsev enega izmed slovenskih zahodnih narec̆ij, ki so prav gotovo glavni posredovalec romanskih izposojenk novejših plasti v slovenski knjižni jezik. 